\title{
Protection of Health Service staff
}

\author{
J. A. LUNN \\ M.D., M.F.C.M., D.I.H. \\ St George's Hospital, London, and Northwick Park Hospital, Harrow, Middlesex
}

\begin{abstract}
Summary
The practical problems involved in immunizing hospital staff are discussed as well as some of the changing priorities for protection as a result of a changing incidence of infectious disease. Some skin problems are mentioned with a view to improving protection for staff at risk. The cost and value of routine stool testing of catering workers is discussed, and the question raised as to whether money saved from these tests might be better spent on improving kitchen hygiene and working facilities.
\end{abstract}

\section{Introduction}

The success of the immunization policies in the past has resulted in such a reduction in the incidence of serious disease that the younger generations in hospital today do not always understand the concern of those with longer memories. When hospitals were small with a total staff of 200 to 300 and the need for protection more evident, adequate immunization of the majority of hospital staff was possible. Today, with district hospitals with up to 3000 staff, it is becoming increasingly difficult to carry out effective and rational protective programmes. Quite often there is more interest in obtaining cholera and typhoid inoculations for holidays abroad than there is for accepting protection from potential infection for the rest of the working year in hospital. Because of the changing patterns of disease it is increasingly necessary to decide how wide a range of staff should be offered protection. The Department of Health recommends that hospital staff in contact with patients should be protected from infectious diseases. There is in fact really no compulsion for staff to accept this protection, and a certain amount of persuasion and education is necessary. The general policy has always been to concentrate on those with close contact with patients or who work in laboratories and postmortem rooms. It is necessary to remember that a far wider range of staff come in contact with patients today than formerly. Nursing duties have been modified so that many aspects of an in-patient's care are now undertaken by non-nursing staff. It is also

Requests for reprints: Dr J. A. Lunn, Northwick Park Hospital, Watford Road, Harrow, Middlesex HA1 3UJ. difficult to segregate staff into risk categories by the nature of the unit they work in because of the frequent interchange of duties. It therefore seems a reasonable policy to offer immunization to all hospital staff, but to concentrate the effort on those more significantly at risk. To detail a list of recommended inoculations would not be of particular interest. There are numerous publications available with this information and a recent issue of The Practitioner (Vol. 215, 1975) provides an excellent symposium entitled 'Immunization: a reappraisal'. What would seem worthwhile is a discussion of the particular practical difficulties encountered when attempting to implement an effective and realistic immunization programme in the context of today's changing patterns of disease.

\section{Smallpox}

Although no longer recommended for infants, vaccination is still advised for hospital staff. With the near extinction of this disease it is a matter of increasing concern to those responsible for protecting hospital staff whether routine vaccination is now justified for all. Where staff have not previously been vaccinated, it would seem more important than ever to recognize contra-indications, and this should include those with past histories of eczema as well as those with the active condition. Of equal importance is the need to advise staff to avoid working in a skin or children's ward until the vaccination lesion has healed if there is any possibility of coming in contact with eczematous patients. It is also necessary to remind staff that they should avoid contact with active eczema cases at home.

Where hospitals have specific isolation units, it is obviously essential to ensure that all staff working in them are protected. The practical problems arise when unprotected staff elsewhere in the hospital are asked to step in at short notice. It is therefore necessary to make sure that there is an adequate reservoir of immunized staff available.

\section{Poliomyelitis}

With such a simple and effective procedure available for complete protection it seems only good 
sense to persuade as many people as possible to accept immunization. For those who have not seen patients in respirators this may seem undue caution, but outbreaks of poliomyelitis are still recorded.

\section{Tuberculosis}

During the course of a year most hospitals have a few cases of open pulmonary tuberculosis admitted to a general ward before a diagnosis is made and a wide variety of staff may be exposed. It is also worth mentioning that tuberculosis is often the commonest serious disease admitted to an isolation unit. It is therefore most important to maintain an effective tuberculin screening programme and B.C.G. (Bacille Calmette-Guérin) given to the susceptible. When an open case has been admitted to a ward, it is essential to have an efficient system for contacting and following-up those who have been exposed. There is invariably some member of staff who has escaped the tuberculin screening programme. Those found to be tuberculin-negative should be removed from exposure and re-tested in 6 weeks' time to confirm that they are still tuberculin negative before being given B.C.G. A follow-up chest X-ray should be arranged 6 months after the initial exposure.

\section{X-rays}

Although tuberculosis is so much reduced in the indigenous population, staff from abroad are more at risk. Screening chest X-rays for new staff should therefore be seen as an important device for protecting staff from this disease. In practice it is often very difficult to ensure that this policy is carried out and close co-operation with the employing authority will be necessary to ensure successful screening.

\section{Rubella}

Staff working in children's wards should be protected. Since there is a rotation of staff between wards, it is difficult to identify those who will be limited to paediatric nursing. Student and pupil nurses will inevitably be exposed during their training and with the increasing trend for earlier marriage, including nurses in training, it is advisable to aim to protect all nursing and other female staff. It is generally recommended that measuring rubella antibody levels should be linked with the initial haemoglobin estimations for new staff. Where hospitals do not have the laboratory facilities for antibody estimation, considerable administrative efforts may be necessary to make arrangements with an outside laboratory. All staff working in antenatal departments should be asked to have their antibody levels estimated and the non-immune protected. Again it is not possible to insist that any given individual has a protective inoculation. How- ever, it is possible to insist that no one works in a maternity unit unless immune from rubella, and this should be the recommended policy.

\section{Tetanus}

It is generally accepted that tetanus toxoid should be given to the general population and hospital staff should be encouraged to accept this protection. Where there are limited resources to carry out this policy, the maximum effort should be concentrated on the higher risk groups, which must include animal house attendants, gardeners, maintenance workers and post-mortem room attendants.

\section{Diphtheria}

When hospital staff are Schick-tested, up to $80 \%$ are found to have protection from previous childhood immunization. The time and expense necessary to identify the susceptible population now seems unjustified, except for staff working in infectious disease or isolation units. The administrative effort and cost necessary to protect even this relatively small group of staff is considerable.

\section{Influenza}

The past enthusiasm for inoculating large groups of workers now seems to be questioned. The cost in materials alone for a large district hospital would be over $£ 3000$. In practice, it is found that only $20 \%$ or less of a hospital population accept influenza immunization. It is probably better to concentrate on those with chronic chest disease, rather than the whole population at random. The recent development of the nasal version of the vaccine has raised interest. It would seem that for optimum protection two attendances are necessary, with the cost being nearly double that of the single injection required for present influenza immunization. Unless the nasal form can be shown to give much better protection there seems no obvious advantage in using it, either financially or administratively.

\section{Serum hepatitis}

The risk of serum hepatitis to staff in renal dialysis units is well known. Outside these units it is difficult to assess the overall risk to hospital staff. The symptomless carrier of the antigen will become a risk when blood specimens are carelessly handled. It is important to emphasize that all hospital staff are potentially at risk if the basic care necessary for dealing with blood samples is neglected and used syringes and needles are inadequately disposed of. Routine care and meticulous technique should eliminate the risk. However, it is important to identify patients who are a source of special risk in the transmission of Australia antigen. Included among these are patients with chronic liver disease 
and those on immunosuppressive therapy. In addition, it is recommended that patients with a history of blood transfusions given abroad and immigrants from African and Asian countries (among whom there may be a $10 \%$ incidence of antigen) should be included in the risk category. When first admitted to hospital these patients should be regarded as a high risk group until blood testing has confirmed otherwise. Those who have positive results should have distinctive yellow labels attached to their notes. Barrier nursing procedures should be employed for taking and handling specimens for laboratory investigation and disposal of excreta and dressings. Blood specimens must be taken only by staff experienced in venepuncture. When blood is taken protective disposable gloves should be worn. The specimen bottles should be appropriately labelled and sent to the laboratory in sealed polythene bags.

In teaching hospitals and research units, staff and students are often asked to give blood for various research projects, or to volunteer for others to practise their venepuncture techniques. It is surprising how little attention is paid to ensuring that staff and students so involved are not antigen carriers, and this is an area where more care should be exercised.

\section{Enteric infection}

Anyone with responsibility for the initial medical screening of food handlers has to decide on the controversial issue of routine stool testing. It is obviously necessary to test the stools of any potential food handler who has a history of enteric disease. The debate centres around those applicants who have no relevant history. The time and expense involved in routine stool testing of this majority may not seem justified by the results or from the evidence provided by many microbiologists. It is accepted that not everyone would agree with this, although there is a general trend towards more selective stool testing. Most would agree, however, with the need to improve the monitoring of hygiene standards amongst hospital canteen staff once employed. The importance of reporting episodes of diarrhoea must be emphasized to every food handler. The fact that many such employees in hospital do not speak English means that they are less likely to be aware of the need to report enteric infections than they should be. Those workers who are aware of the need to stop handling food when suffering with enteric symptoms may sometimes be reluctant to report sick for fear of loss of earnings. It is important to make sure that enforced absence from work in order to protect others from potential disease does not result in loss of income for those who accept this discipline.

Where non-food handlers are concerned, it is necessary to have a policy for stool testing which is not only rational, but which at the same time will not $\frac{3}{\infty}$ deter staff from reporting their symptoms. If all staff $\frac{\varrho}{c}$ who report episodes of diarrhoea are requested to $\stackrel{.}{\Rightarrow}$ send stool specimens regardless of the significance $\stackrel{\vec{\rho}}{\rightarrow}$ of the history or symptoms, there is a tendency for $\bar{c}$ staff to fail to report their condition because of a general reluctance to send stool specimens. For this reason it is important to be selective in requesting stool specimens. Where this is so, there is much more likelihood that episodes of diarrhoea will be reported, and the pattern throughout the hospital known. Quite clearly, when enteric infection is known to be present in a ward, much closer attention must be paid to symptoms. The need to co-operate with the microbiology department cannot be too strongly stressed.

A further point may be worth making. It is now abundantly evident that financial resources for hospitals are strictly limited. The value obtained from what is spent must be increasingly critically assessed. Many of our older hospitals have kitchens which are physically inadequate for optimum hygiene. Washing facilities may be poor and too far removed from the place of work. These poor environmental conditions may be the most important factors in producing low standards of hygiene and potential infection. The cost of one stool test is at least $\mathrm{f4}$. It may well be better to direct money fron non-essential laboratory tests to providing muck needed improvements to many catering areas in ouf hospitals.

\section{Skin infections \\ Herpetic whitlow}

These lesions are usually seen among nursing staff who have been handling upper respiratory-tract secretions containing the virus. Postoperative care of neurosurgical patients is a common source of infection, and intensive care units present a similar situation. A typical lesion begins as a painful deepseated vesicle, which later becomes more superficial and raised, with some tissue destruction. Small deep vesicles beyond the edge of the lesion are characteristic of the infection. It is important to recognize the condition because resolution will occur without specific treatment, and incision should be avoided. Where practical, nursing staff should wear protective gloves when at risk from handling upper respiratorytract secretions, and this precaution should not be confined only to staff in neurosurgical and intensive care units.

\section{Chronic paronychia}

A low-grade but persistent form of paronychia occurs in nurses. This infection may be difficult to eradicate. Trauma often from repeated scrubbing up seems the commonest aggravating factor, so that the skin surface around the nail-base becomes cracked or 
fissured. A poor peripheral circulation may accompany the condition. Unless the sufferer is removed from the predisposing situation little improvement occurs. Sometimes Candida organisms may be associated with the problem.

Because infection may remain beneath the base of the nail, often a recurrence is preventable only by removal of the nail and by local antibiotic treatment.

\section{Scabies}

There has been a marked increase in the incidence and prevalence of this condition in recent years. Changing social customs probably account for this increase. Among certain individuals there has been a trend for less conventional styles of living, where previously accepted standards of hygiene have been discarded. The overcrowding involved in the new modes of existence, together with the lack of hygiene, has undoubtedly predisposed to the scabies increase. Unfortunately, the condition often goes unrecognized in patients admitted to hospital for general medical or surgical conditions. Staff who come into direct contact with patients are at risk, and it is now not at all uncommon for nurses, radiographers and physiotherapists to acquire the condition. It is necessary to make all staff more aware of the possibility of scabies being present in patients, and when patients are admitted to wards closer inspection of the characteristic areas of skin involvement is advisable.

\section{Reference}

The Practitioner (1975) Symposium on Immunization: A Reappraisal. The Practitioner, 215. 